\title{
The voltage dependence of hEag currents is not determined solely by membrane-spanning domains
}

\author{
Éva Lörinczi · Joanna Napp · \\ Constanza Contreras-Jurado • \\ Luis A. Pardo · Walter Stühmer
}

Received: 30 January 2008/Revised: 17 March 2008/Accepted: 18 March 2008/Published online: 1 April 2008

(C) The Author(s) 2008

\begin{abstract}
The ether-à-go-go potassium channels hEag1 and hEag2 are highly homologous. Even though both possess identical voltage-sensing domain S4, the channels act differently in response to voltage. Therefore we asked whether transmembrane domains other than the voltage sensor could contribute to the voltage-dependent behaviour of these potassium channels. For this chimaeras were created, in which each single transmembrane domain of hEag1 was replaced by the corresponding segment of hEag2. The voltage-dependent properties of the chimaeras were analysed after expression in Xenopus laevis oocytes using the two-electrode voltage-clamp method. By this we found, that only the mutations in transmembrane domains S5 and S6 are able to change the voltage sensitivity of hEag 1 by shifting the half-activation potential $\left(V_{50}\right)$ to values intermediate between the two wild types. Moreover, the presence of $\mathrm{Mg}^{2+}$ has strong effects on the voltage sensitivity of hEag2 shifting $V_{50}$ by more than $50 \mathrm{mV}$ to more positive values. Interestingly, despite the identical binding site $\mathrm{Mg}^{2+}$ showed only little effects on hEag1 or the chimaeras. Altogether, our data suggest that not only transmembrane spanning regions, but also non-membrane spanning regions are responsible for differences in the behaviour of the hEag1 and hEag2 potassium channels.
\end{abstract}

EBSA Satellite meeting: Ion channels, Leeds, July 2007.

É. Lörinczi · J. Napp · C. Contreras-Jurado ·

L. A. Pardo $(\square)$. W. Stühmer

Max-Planck Institute of Experimental Medicine,

Hermann-Rein Str. 3, 37075 Göttingen, Germany

e-mail: pardo@em.mpg.de

\section{Introduction}

Ether-à-go-go (Eag) channels belong to the one of the latest described members of the voltage-gated potassium channel $\left(K_{v}\right)$ family, the Eag $\left(K_{v} 10\right)$ subfamily. Sequence alignments of Drosophila, rat, mouse and human have shown that they are structurally related not only to the Shaker family of voltage-gated potassium channels but also to the cyclic nucleotide-gated channels. Similar to other $K_{v}$ channels, the functional Eag channel is assumed to be a tetramer, each monomer consisting of six putative transmembrane domains, with the $\mathrm{S} 4$ domain serving as the main voltage sensor. In addition, they also possess long intracellular N- and C-termini (Bauer and Schwarz 2001; Yellen 2002).

The human genome has two isoforms of Eag channels, hEag1 (KCNH1, $\left.\mathrm{K}_{\mathrm{v}} 10.1\right)$ and hEag2 (KCNH5, $\left.\mathrm{K}_{\mathrm{v}} 10.2\right)$. Both are expressed mainly in the central nervous system, although hEag2 has also been found in skeletal muscle, heart, lung, liver, placenta, kidney and pancreas (Ju and Wray 2002; Schonherr et al. 2002a). Based on their biophysical properties (outward-rectification, lack of inactivation and the dependence of activation on the holding potential), determined using heterologous expression systems, both proteins are assumed to be involved in setting of the membrane potential, modulating action potential duration and firing frequency of excitable cells. So far, endogenous currents have only been measured from hEag 1 in cultured myoblasts and tumour cells, but have not been reported from neurons. hEag1 has also been correlated to processes like cell-cycle and proliferation, and appears to be implicated in tumour progression in up to $75 \%$ of solid tumours. (Bauer and Schwarz 2001; Hemmerlein et al. 2006; Pardo 2004). In contrast, hEag2, besides being ubiquitous, does not have the oncogenic potential of hEag1. 
Although the two proteins are $73 \%$ identical, they significantly differ in their voltage sensitivity and activation kinetics; hEag2 activates at more hyperpolarised potentials than hEag1, shows a shallower voltage-dependence and a less dramatic influence of the holding potential on the activation time constant compared to hEag1 (Ju and Wray 2002; Schonherr et al. 2002a). Ju and Wray extensively studied the activation properties employing chimaeras of the two hEag isoforms, exchanging the amino- and carboxy-termini and blocks of the membrane-spanning parts of the proteins. They concluded that the activation involves several parts of the protein, ranging from the $\mathrm{N}$-terminus to various segments of the transmembrane domains $(\mathrm{Ju}$ and Wray 2006). By swapping the entire membrane-spanning region between hEag1 and hEag2 they also deduce that the whole membrane-spanning region behaves as a functional unit. We decided to test how each of the Eag2 transmembrane segments behaves in the context of the Eag1 background.

\section{Materials and methods}

\section{Molecular biology}

For chimera construction, the starting clones were wildtype hEag1 and hEag2 in the pSGEM oocyte expression vector (a gift from M. Hollmann, Bochum). Chimaeras of hEag1, with transmembrane segments replaced by the corresponding ones of hEag2, were constructed by replacing the differing amino acids using site-directed mutagenesis (QuickChange Kit, Stratagene). The constructs were named: h1.S1(h2) (V215A, I223V, L234M); h1.S2(h2) (V253L, I256L); h1.S3(h2) (V308I); h1.S5(h2) (A362V, M366L); h1.S6(h2) (I451M, A454S, I456M, I459V), where Sx stands for the TM segment of hEag1 (h1) replaced by corresponding region of hEag2 (h2). The fourth transmembrane domain (S4) is identical in both proteins.

After linearisation with SfiI, cRNA was prepared in vitro using the T7 promoter contained in the pSGEM vector, with the T7 mMessage mMachine kit (Ambion). Oocytes were injected with $0.025-1 \mathrm{ng}$ cRNA (hEag1 and chimaeras) (hEag1 and the chimaeras) or 12.5-25 ng cRNA (hEag2) and kept at $18^{\circ} \mathrm{C}$ in $\mathrm{ND} 96$ solution $(96 \mathrm{mM} \mathrm{NaCl}$, $2 \mathrm{mM} \mathrm{KCl}, 0.2 \mathrm{mM} \mathrm{CaCl} 2,2 \mathrm{mM} \mathrm{MgCl}_{2}, 0.5 \mathrm{mM}$ teophylline, $5 \mathrm{mM}$ HEPES, $\mathrm{pH}$ 7.5).

\section{Electrophysiology}

Two-electrode voltage-clamp recordings were performed 1-3 days after cRNA injection, using a Turbo TEC-10CD amplifier (NPI electronics) at room temperature. The intracellular electrodes had resistances of $0.3-1.5 \mathrm{M} \Omega$ when filled with $2 \mathrm{M} \mathrm{KCl}$. The extracellular measuring solution (Normal Frog Ringer, NFR) contained $115 \mathrm{mM}$ $\mathrm{NaCl}, 2.5 \mathrm{mM} \mathrm{KCl}, 1.8 \mathrm{mM} \mathrm{CaCl}, 10 \mathrm{mM} \mathrm{HEPES/}$ $\mathrm{NaOH}, \mathrm{pH} 7.2$, with or without $5 \mathrm{mM} \mathrm{MgCl}_{2}$.

Data acquisition and analysis were performed with the Pulse-PulseFit (HEKA Electronics) and IgorPro (WaveMetrics) software packages. Current records were sampled at $1 \mathrm{kHz}$. The cells were held at $-100 \mathrm{mV}$ membrane potential. The applied voltage protocols are described in the figure legends. No leak current subtraction was carried out.

Voltage dependence of activation was estimated by fitting the current-voltage relationships measured from individual cells according to a Hodgkin-Huxley formalism (Terlau et al. 1997) (Eq. 1) taking also into account the rectification at positive potentials where necessary $(\mathrm{Eq} 2)$ :

$I(V)=\Gamma \frac{\left(V-V_{\text {rev }}\right)}{\left(1+e^{\left(\left(V_{50}-V\right) / k\right)}\right)^{4}}$
$I(V)=\Gamma \frac{\left(V-V_{\text {rev }}\right)}{\left(1+e^{\left(\left(V_{50}-V\right) / k\right)}\right)^{4}}-\frac{1}{1+e^{\left(\left(V_{50 \mathrm{~B}}-V\right) / k_{\mathrm{B}}\right)}}$

where $\Gamma$ is the total conductance, $V_{\text {rev }}$ the reversal potential [fixed at the value of $-98.5 \mathrm{mV}\left(\mathrm{Ju}\right.$ and Wray 2002)], $V_{50}$ the potential of half activation per subunit and $k$ the slope factor; similarly the parameters $V_{50 \mathrm{~B}}$ and $k_{\mathrm{B}}$ characterise the rectification at positive potentials.

\section{Results and discussion}

Current-voltage relationship in the absence of external magnesium

Wild-type hEag1, hEag2 and their chimaeras h1.S1(h2), h1.S2(h2), h1.S3(h2), h1.S5(h2), h1.S6(h2) were expressed in Xenopus oocytes and their activity was assessed by means of two-electrode voltage-clamp. Schönherr et al. (2002a) reported lower expression levels of hEag2 compared to hEag1. We were therefore especially careful with the expression constructs, which differ exclusively in the open reading frame of the channel. Still, comparable amplitudes of hEag1 and hEag2 WT currents could only be obtained when the concentration of injected cRNA for hEag2WT was at least 20 times higher. Expression levels of all the chimaeras were very similar to hEag1WT.

It is noteworthy that hEag 1 shows rectification at very positive potentials, whereas hEag2 does not. This aspect had to be taken into account in order to optimise the range of potentials used and similar parameters. Therefore, we used Eq. 2 whenever an inward rectification was evident, otherwise Eq. 1 was used. Since rectification can be attributed to 
block by intracellular sodium (Pardo et al. 1998), it can (and does) vary from oocyte to oocyte. Fitting the parameters of Eq. 1 or Eq. 2 allowed reasonable fits to the average normalised current values for all oocytes recorded.

The voltage dependence of each chimera in the absence of $\mathrm{Mg}^{2+}$ was first compared to those of the original wild types (Fig. 1).

Regardless of the transmembrane domain replaced in hEag1, the behaviour of all chimaeras was closer to hEag1 than to hEag2. The most prominent differences were observed in h1.S5(h2) and h1.S6(h2), where the halfpotentials of activation lay between those of the wild types, although still closer to hEag1. A slight shift in the halfactivation potential was also noticeable with h1.S1(h2). Interestingly, the slope factors of all (including S5 and S6) mutants were very similar to that of hEag1WT.

These data suggest that the residues of transmembrane domains S5 and S6 (which form the pore-domain) could contribute to the activation threshold of hEag channels, since mutants h1.S5(h2) and h1.S6(h2), open at potentials more negative compared to hEag1. However, all the transmembrane segments seem to play only a small role in the differences in the voltage-sensitivity between the two wild-type channels, since the slope factor of all mutants is very similar to that of hEag1.

$\mathrm{Ju}$ and Wray (2006) in their extensive study using chimaeras of hEag1 and hEag2 have already indicated the involvement of the entire membrane-spanning region in the steady-state activation properties of the hEag channels. However, they used constructs where larger segments were replaced (regions S1-S3 and S5-S6 of hEag2 introduced into hEag1), and did not investigate the role of single transmembrane domains. Our results support and complement their findings, more specifically, the idea that the kinetics of steady-state activation depends on multiple domains within or/and between channel subunits. How important other parts of the sequence are, still needs to be investigated, but the involvement of the N-termini has already been shown for the human Eag 1 and Eag2, and rat Eag1 isoforms (Ju and Wray 2006; Terlau et al. 1997).

Current-voltage relationship in the presence of external magnesium

Extracellular $\mathrm{Mg}^{2+}$ induces strong modifications in the activation time constants of hEag1 and hEag2, which have been extensively investigated for various isoforms of Eag1WT and to less extent for hEag2WT (Ju and Wray 2002; Schonherr et al. 2002a; Silverman et al. 2000; Tang et al. 2000; Terlau et al. 1996).

Addition of $5 \mathrm{mM} \mathrm{Mg}^{2+}$ to the extracellular medium led to a positive shift of $V_{50}$ and a slightly steeper voltagedependence in all channels (Fig. 2).
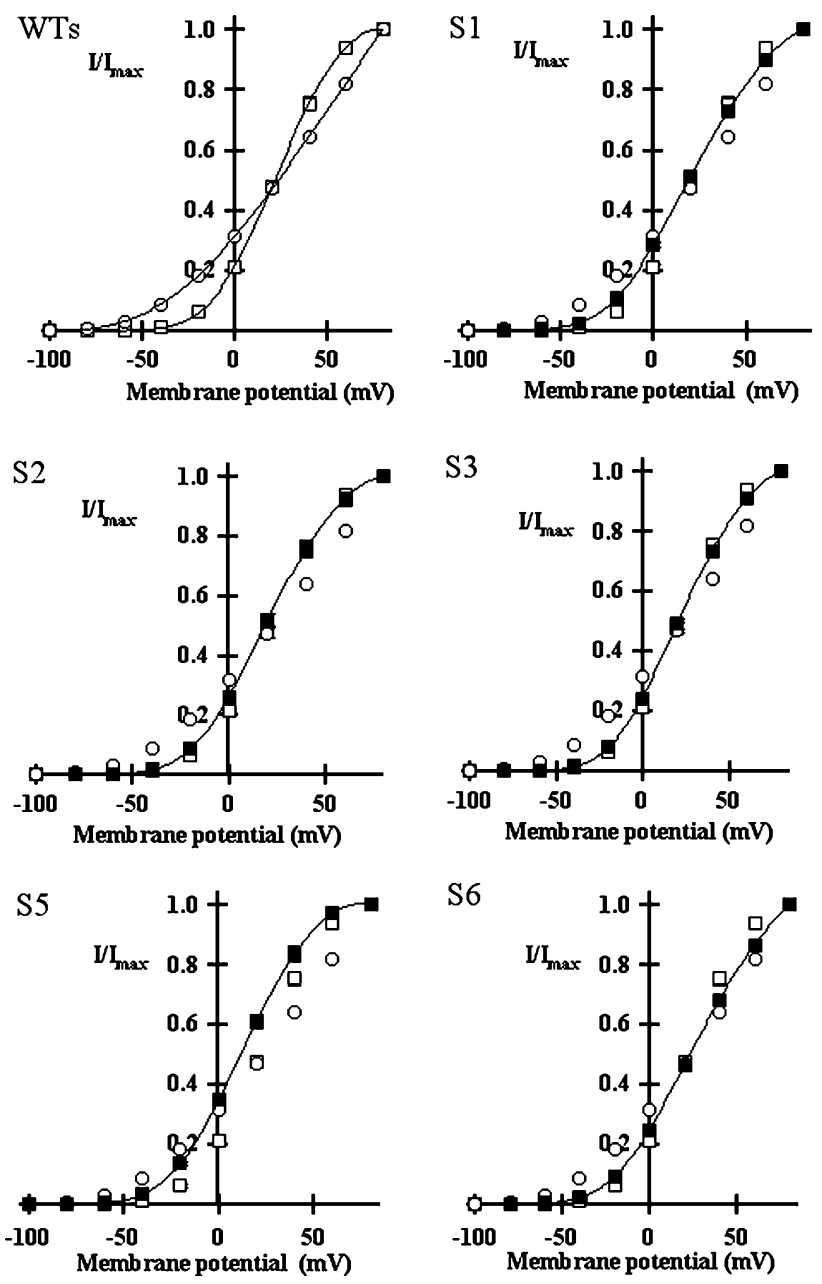

Fig. 1 Current-voltage relationships in the absence of $\mathrm{Mg}^{2+}$. I-V relationships were determined by measuring the whole cell current triggered from a holding potential of $-100 \mathrm{mV}$ and stepping to a test potential from $-100 \mathrm{mV}$ to $+80 \mathrm{mV}$ for $500 \mathrm{~ms}$ in $20 \mathrm{mV}$ increments. The time interval between two consecutive test pulses was $17 \mathrm{~s}$. The current amplitude was measured at the end of the test pulse and normalised to the value recorded at $+80 \mathrm{mV}\left(I / I_{\max }\right)$ and plotted against the applied membrane potential. WTs: hEag1 and hEag2 wild types shown alone; S1-S6: chimaeras compared to both wild types. Symbols: open square hEag1 WT $(n=25)$, open circle hEag2 WT $(n=34)$, filled square chimaera $[S 1(n=22) . S 2(n=22), S 3(n=30), S 5$ $(n=25)$, $S 6(n=22)]$. Values are shown in mean \pm SEM. The continuous lines represent fits of the data points by Eqs. 1 or 2

The effect on hEag2WT was the most intense, by more than $50 \mathrm{mV}$, and had not been previously reported. In the presence of $\mathrm{Mg}^{2+}$, the biophysical properties of hEag2 are very similar to those of hEag1. We do not think that such a strong right-shift in the half-activation potential can be explained solely through a surface charge effect, since the effects on other channels have a much more reduced magnitude. It is important to point out that this property of hEag2 might be of importance under physiological conditions $\left(2 \mathrm{mM} \mathrm{Mg}^{2+}\right)$, where the only likely difference between 

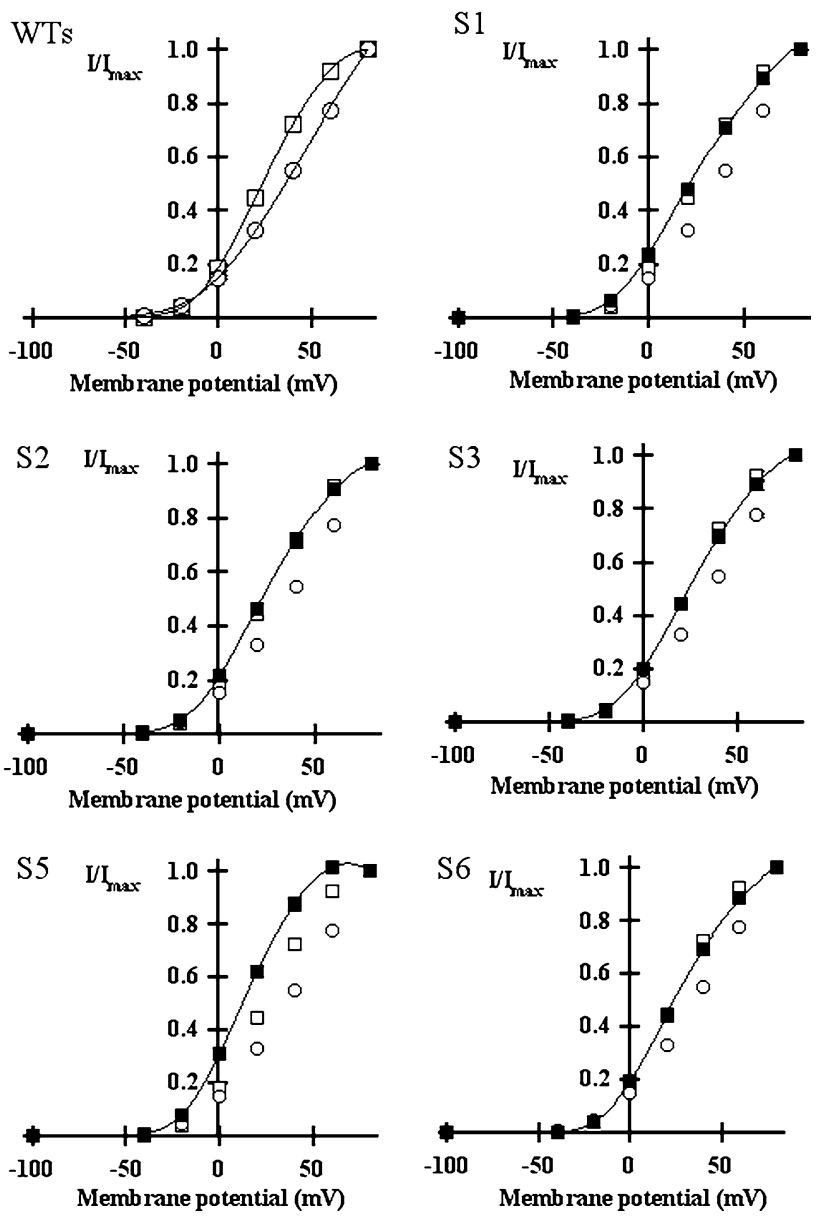

Fig. 2 Current-voltage relationships in the presence of $5 \mathrm{mM} \mathrm{Mg}^{2+}$. The same pulse protocol applied as in the absence of $\mathrm{Mg}^{2+}$, with difference in the duration of the test pulse $(1 \mathrm{~s}$ instead of $500 \mathrm{~ms})$. WTs: hEag1 and hEag2 wild types shown alone; $S 1-S 6$ : chimaeras compared to both wild types. Again, the various constructs are represented by: open square hEag1 WT $(n=22)$, open circle hEag2 WT $(n=21)$, filled square chimaera $[S 1(n=20) . S 2(n=16), S 3(n=18), S 5$ $(n=14)$, $S 6(n=13)]$. Values are shown in mean \pm SEM. The continuous lines represent fits of the data points by Eqs. 1 or 2

hEag1 and hEag2 in electrophysiological terms would be the slope of the I-V relationship, and not any longer the activation threshold.
The positive shift in of the activation threshold was evident, although less prominent in hEag1WT and the mutant chimaeras containing the $\mathrm{S} 1, \mathrm{~S} 2$ and $\mathrm{S} 3$ segments of hEag2. Again the S5 and S6 chimaeras showed a more pronounced effect, intermediate between wild-type hEag1 and hEag2, although closer to hEag1.

The current-voltage parameters of all mutants are summarised in Table 1.

These data suggest that $\mathrm{Mg}^{2+}$ affects especially the activation-threshold of the proteins (i.e. contributes to the reduction of open probability at negative potentials), and lessen their voltage sensitivity. Our observations are compatible with those reported for the Drosophila channels (Silverman et al. 2000).

The rectification of wild-type hEag1 has been related to block by intracellular sodium, regulated by cytoskeletal interactions (Camacho et al. 2000). This effect had not been reported for hEag2. Although the buffer where the oocytes were placed after cRNA injection contains theophyllin in order to impair their maturation further, hEag1WT, S5 and S6 displayed rectification at positive potentials in all or most of the measured oocytes. Not surprisingly for mutation in transmembrane domains, none of the chimaeras showed a complete lack of rectification similar to hEag2. Interestingly, the rectification was clearly intensified in the S5 chimaera, indicating an implication of this segment (or the S4-S5 linker) in this property.

Dependence of channel activation on the holding potential

The hallmark of Eag currents is their strong dependence of activation kinetics on holding potential, reminiscent of the so-called "Cole-Moore shift" described for potassium currents in the squid axon (Cole and Moore 1960). This aspect was investigated in all constructs, in the absence and presence of $5 \mathrm{mM} \mathrm{Mg}^{2+}$, and the results are summarised in Figs. 3 and 4. Again, as a general observation, the mutant channels behave similar to hEag1WT, and very different from hEag2, both in the absence and presence of $\mathrm{Mg}^{2+}$.
Table 1 Summary of parameters from fits

\begin{tabular}{lccccc}
\hline & \multicolumn{2}{l}{$0 \mathrm{Mg}$} & & $5 \mathrm{Mg}$ & \\
\cline { 2 - 3 } \cline { 5 - 6 } & $\mathrm{V}_{50}(\mathrm{mV})$ & $k(\mathrm{mV})$ & & $V_{50}(\mathrm{mV})$ & $k(\mathrm{mV})$ \\
\hline hEag1 WT & $-24.2 \pm 6.6$ & $25.6 \pm 2.5$ & & $-21.76 \pm 4.4$ & $23.67 \pm 2.1$ \\
hEag2 WT & $-79.4 \pm 8.6$ & $50 \pm 6$ & & $-27 \pm 6.7$ & $33.8 \pm 4.42$ \\
h1.S1(h2) & $-36 \pm 9.75$ & $26.1 \pm 3.6$ & & $-31.5 \pm 5.75$ & $24.65 \pm 3.5$ \\
h1.S2(h2) & $-33.78 \pm 4.3$ & $26.45 \pm 3.5$ & & $-28 \pm 5$ & $24 \pm 2.7$ \\
h1.S3(h2) & $-33.7 \pm 8.8$ & $27.5 \pm 4.7$ & & $-26 \pm 8.7$ & $24.55 \pm 5$ \\
h1.S5(h2) & $-42.6 \pm 7.5$ & $28.2 \pm 4.1$ & & $-29.1 \pm 4.1$ & $21 \pm 1.52$ \\
h1.S6(h2) & $-40 \pm 8.2$ & $29.73 \pm 4.53$ & & $-25.3 \pm 4$ & $23.87 \pm 2.3$ \\
\hline
\end{tabular}



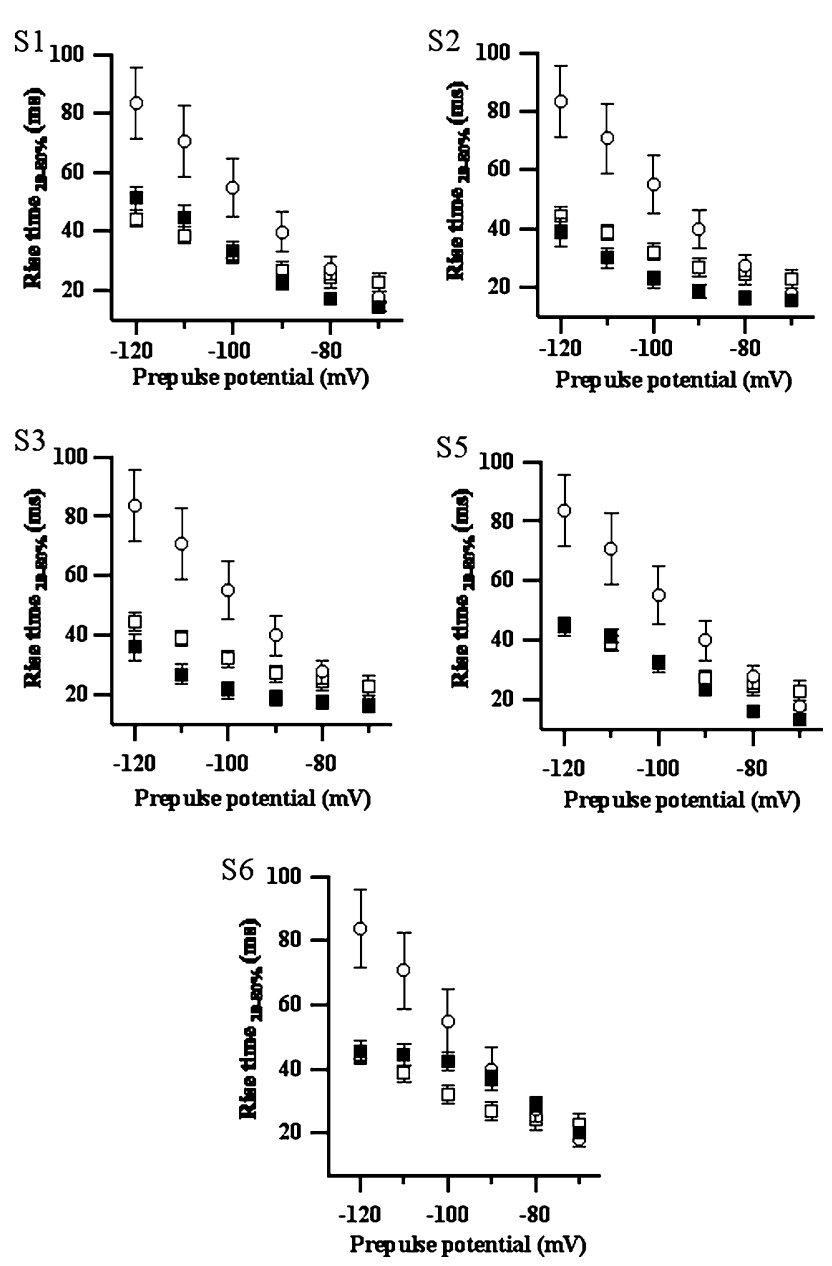

Fig. 3 Dependence of activation on the holding potential in the absence of $\mathrm{Mg}^{2+}$. The pulse protocol was a $500 \mathrm{~ms}$ depolarisation to $+40 \mathrm{mV}$ preceded by 5 -s prepulses at potentials ranging from -120 to $-70 \mathrm{mV}$ in $10 \mathrm{mV}$ increments. The time interval between two consecutive test-pulses was $20 \mathrm{~s}$. In each case the rise time of activation (time for $20-80 \%$ of maximal current $=\tau_{20-80 \%}$ ) was determined (Ju and Wray 2002), plotted against the values of holding potential, and each mutant compared separately with both wild types (S1-S6). Symbols: open square hEag1 WT $(n=18)$, open circle hEag2 WT $(n=32)$, filled square chimaera $[S 1 \quad(n=24)$. $S 2$ $(n=22), S 3(n=27), S 5(n=23), S 6(n=22)]$. Values are shown in mean \pm SEM

In the absence of $\mathrm{Mg}^{2+}$, the voltage dependence was steeper for hEag2 than for hEag1; at more negative potentials the activation of hEag2 is twice as slow compared to hEag1, but the differences were minimised at $70 \mathrm{mV}$. The activation of the chimaeras resembled that of hEag1WT, being even faster in case of S2 and S3. In terms of voltage sensitivity, S1 and S5 gained and S6 lost voltage dependence compared to hEag1. Also, at prepulse membrane potentials positive to $-90 \mathrm{mV}, \quad \mathrm{S} 6$ and $\mathrm{hEag} 2$ activated in a similar manner.

Interestingly, at membrane potentials where hEag2 channels start to open, the acceleration of activation with
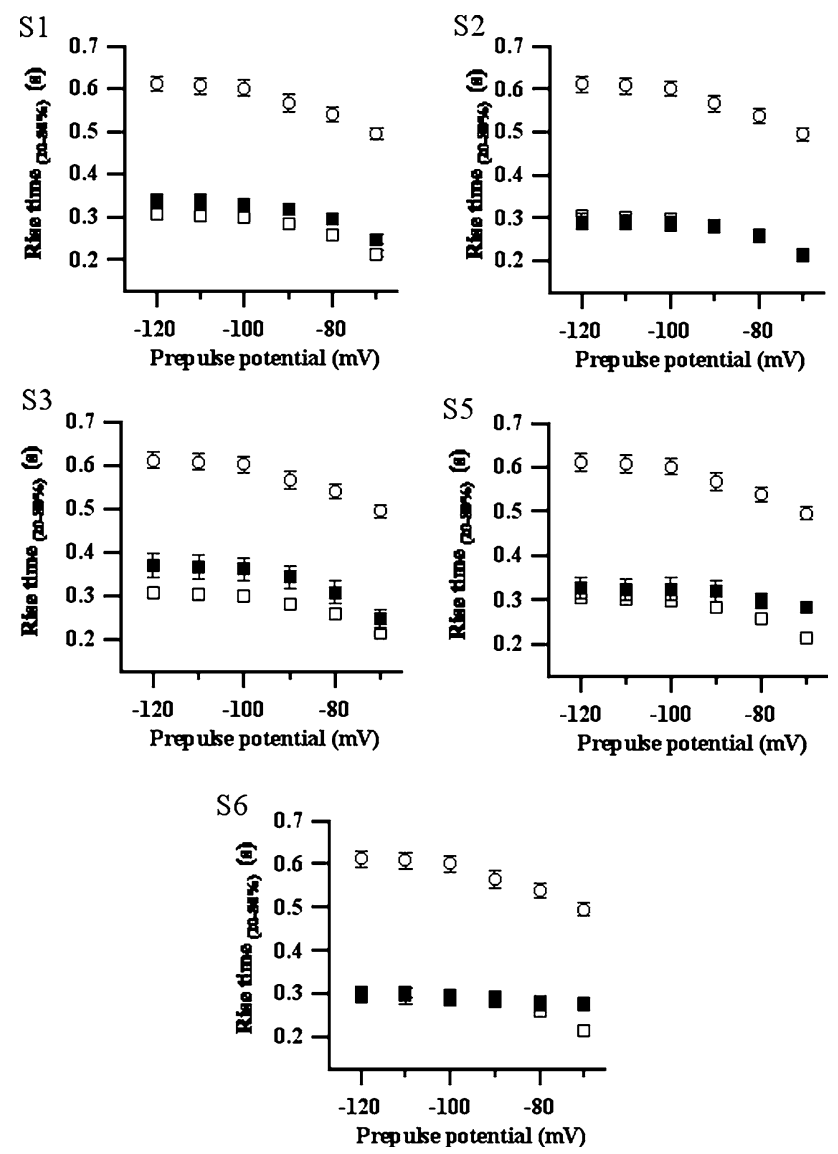

Fig. 4 Dependence of activation on the holding potential in the presence of $5 \mathrm{mM} \mathrm{Mg}^{2+}$. The same pulse protocol was applied as in Fig. 3, except that the depolarisation to $+40 \mathrm{mV}$ lasted $1 \mathrm{~s}$, to allow the currents to reach steady-state activity. Again, in each case the rise time of activation was determined, plotted against the values of holding potential, and each mutant compared separately with both wild types (S1-S6). Symbols: open square hEag1 WT $(n=17)$, open circle hEag2 WT $(n=14)$, filled square chimaera [S1 $(n=16) . S 2$ $(n=16), S 3(n=20), S 5(n=15), S 6(n=14)]$. Values are shown in mean \pm SEM

prepulse potential kept the same slope, although a contribution of open channels at the prepulse potential would be expected. This observation suggests that channel activation is less dependent on membrane potential than assumed so far (Ju and Wray 2006; Schonherr et al. 2002b).

Addition of $5 \mathrm{mM} \mathrm{Mg}^{2+}$ strongly slowed down the time course of activation; both hEag1 and hEag2 became $8-10$ times slower and less sensitive to negative potentials. As in the absence of $\mathrm{Mg}^{2+}$, hEag1 was twice as fast as hEag2. The chimaeras again resembled hEag1WT, with the difference that S5 and S6 lost voltage dependence over the tested range, suggesting that $\mathrm{Mg}^{2+}$ impairs voltage-dependent conformational changes in the latter constructs.

Silverman et al. have shown that $\mathrm{Mg}^{2+}$ binds to aspartate residues within transmembrane segments 2 and 3 of the Drosophila channel (Silverman et al. 2000). However, all 
Asp residues are conserved between hEag1 and hEag2, and consequently the binding site for $\mathrm{Mg}^{2+}$ should be conserved in all reported constructs. Therefore we rather expect the link between divalent bonding and gating mechanism to be altered and not the binding of $\mathrm{Mg}^{2+}$. On one hand, there is a dramatic change in the half-activation potential of hEag2WT in spite of the shared Asp residues; on the other hand introduction of the transmembrane domains of hEag2WT into hEag1WT does not change the hEag1-like behaviour of the mutants. Therefore, the $\mathrm{N}$ and/or C-termini of the channels should modulate the behaviour of the channel core after $\mathrm{Mg}$ is bound, and contribute to the overall effect of $\mathrm{Mg}$.

To conclude, our data suggest that voltage dependence in the hEag channels is not determined by any one of the transmembrane domains alone, since the behaviour of all mutants resembles that of hEag1WT. However, transmembrane domains S5 and S6 contribute to defining the activation threshold, because these chimaeras activate at membrane potentials intermediate between the two wild types. In addition, we also report the strong effect of $\mathrm{Mg}^{2+}$ on the activation threshold of hEag2WT, and almost none on that of hEag1WT and the chimaeras, meaning that next to the ion-binding site, the overall effect of $\mathrm{Mg}^{2+}$ is mediated by other parts of the proteins.

Open Access This article is distributed under the terms of the Creative Commons Attribution Noncommercial License which permits any noncommercial use, distribution, and reproduction in any medium, provided the original author(s) and source are credited.

\section{References}

Bauer CK, Schwarz JR (2001) Physiology of EAG $\mathrm{K}^{+}$channels. J Membr Biol 182:1-15
Camacho J, Sánchez A, Stühmer W, Pardo LA (2000) Cytoskeletal interactions determine the electrophysiological properties of human EAG potassium channels. Pflugers Arch 441:167-174

Cole K, Moore J (1960) Potassium ion current in the squid giant axon: dynamic characteristic. Biophys J 1:1-14

Hemmerlein B, Weseloh RM, Mello de Queiroz F, Knötgen H, Sánchez A, Rubio ME, Martin S, Schliephacke T, Jenke M, Radzun HJ , Stühmer W, Pardo LA (2006) Overexpression of Eag1 potassium channels in clinical tumours. Mol Cancer 5:41. doi:10.1186/1476-4598-5-41

Ju M, Wray D (2002) Molecular identification and characterisation of the human eag2 potassium channel. FEBS Lett 524:204-210

Ju M, Wray D (2006) Molecular regions responsible for differences in activation between heag channels. Biochem Biophys Res Commun 342:1088-1097

Pardo LA (2004) Voltage-gated potassium channels in cell proliferation. Physiology 19:285-292

Pardo LA, Brüggemann A, Camacho J, Stühmer W (1998) Cell cyclerelated changes in the conducting properties of r-eag $\mathrm{K}^{+}$ channels. J Cell Biol 143:767-775

Schonherr R, Gessner G, Lober K, Heinemann SH (2002a) Functional distinction of human EAG1 and EAG2 potassium channels. FEBS Lett 514:204-208

Schonherr R, Mannuzzu LM, Isacoff EY, Heinemann SH (2002b) Conformational switch between slow and fast gating modes: allosteric regulation of voltage sensor mobility in the EAG $\mathrm{K}^{+}$ channel. Neuron 35:935-949

Silverman WR, Tang C-Y, Mock AF, Huh K-B, Papazian DM (2000) $\mathrm{Mg}^{2+}$ modulates voltage-dependent activation in ether-a-go-go potassium channels by binding between transmembrane segments S2 and S3. J Gen Physiol 116:663-678

Tang C-Y, Bezanilla F, Papazian DM (2000) Extracellular $\mathrm{Mg}^{2+}$ modulates slow gating transitions and the opening of Drosophila ether-a-go-go potassium channels. J Gen Physiol 115:319-338

Terlau H, Ludwig J, Steffan R, Pongs O, Stühmer W, Heinemann SH (1996) Extracellular $\mathrm{Mg}^{2+}$ regulates activation of rat eag potassium channel. Pflugers Arch 432:301-312

Terlau H, Heinemann SH, Stuhmer W, Pongs O, Ludwig J (1997) Amino terminal-dependent gating of the potassium channel rat eag is compensated by a mutation in the S4 segment. J Physiol 502:537-543

Yellen G (2002) The voltage-gated potassium channels and their relatives. Nature 419:35-42 